Relations industrielles

Industrial Relations

\title{
Wayne LEWCHUK : American Technology and the British Vehicle Industry. Cambridge, Cambridge University Press, 1987, 304 pp., ISBN 0521-30269-2
}

\section{Jacques Bélanger}

Volume 43, numéro 3, 1988

URI : https://id.erudit.org/iderudit/050445ar

DOI : https://doi.org/10.7202/050445ar

Aller au sommaire du numéro

Éditeur(s)

Département des relations industrielles de l'Université Laval

ISSN

0034-379X (imprimé)

1703-8138 (numérique)

Découvrir la revue

Citer ce compte rendu

Bélanger, J. (1988). Compte rendu de [Wayne LEWCHUK : American Technology and the British Vehicle Industry. Cambridge, Cambridge University Press, 1987, 304 pp., ISBN 0521-30269-2]. Relations industrielles / Industrial Relations, 43(3), 716-717. https://doi.org/10.7202/050445ar

Tous droits réservés @ Département des relations industrielles de l'Université Laval, 1988
Ce document est protégé par la loi sur le droit d'auteur. L’utilisation des services d’Érudit (y compris la reproduction) est assujettie à sa politique d'utilisation que vous pouvez consulter en ligne.

https://apropos.erudit.org/fr/usagers/politique-dutilisation/ 
American Technology and the British Vehicle Industry, par Wayne Lewchuk, Cambridge, Cambridge University Press, 1987, 304 pp., ISBN 0521-30269-2

Wayne Lewchuk, professeur à McMaster University et spécialiste de l'histoire économique, présente une étude très documentée sur l'histoire de l'industrie automobile britannique. La recherche porte principalement sur la période 1890-1930, durant laquelle s'est développé ce que l'auteur appelle le système britannique de production de masse, une alternative au modèle mis de l'avant par Henry Ford. Car, comme le suggère le titre, l'auteur élabore sa thèse sur la base d'une comparaison fort pertinente avec les États-Unis, ce qui ajoute à l'intérêt des lecteurs nord-américains. Bien que les matériaux présentés portent surtout sur la dite période, un dernier chapitre applique le modèle d'analyse à la période contemporaine, jusqu'aux années 1980 .

Selon son champ disciplinaire et sa spécialité, le lecteur y trouvera des apports différents. Nous ferons état de trois thèmes particulièrement intéressants, à savoir la formulation théorique sur le contrôle du travail, l'origine du fordisme aux États-Unis, et la plus récente crise de la production automobile en Grande-Bretagne.

L'auteur se situe dans le courant de l'analyse matérialiste du processus de production et centre la discussion sur le problème de la conversion de la force de travail en effort dans le processus de production. Alors que l'étude économique du progrès technologique porte le plus souvent sur le ratio capital-travail et le coût de ces facteurs de production, Lewchuk est davantage préoccupé par l'utilisation plus ou moins efficace de ces mêmes intrants. Du rapport d'achat et de vente de la force de travail, l'attention se déplace vers la question du «control over effort norms». Sur la base de matériaux historiques, l'auteur étudie l'interaction complexe entre le rapport rémunération-effort («effort bargain») et le processus de génération de la technologie.

On retient ensuite un remarquable chapitre sur l'origine du fordisme, l'étude portant notamment sur l'implantation de la chaîne d'assemblage mécanisée à la Ford Motor Company en 1913. L'auteur montre les implications considérables de cette innovation sur la productivité et le contrôle managérial. Il situe ensuite ce développement technologique par rapport au contexte institutionnel dans lequel évoluait alors le processus de production (l'auteur introduit le concept de «production institutions»). La stratégie fordiste à l'état pur, incluant une hausse de salaires substantielle, s'inscrivait dans le développement d'un nouveau modèle de contrôle du travail, nécessité dans une bonne mesure par la forte croissance de l'entreprise et la résistance ouvrière. Appuyant les thèses de Lichtenstein et de Herding, l'auteur argumente que les travailleurs de l'automobile ont alors renoncé à leur discrétion sur l'utilisation de leur force de travail, sans jamais pouvoir la reprendre par la suite. Selon Lewchuk, bien que la domination de la chaîne mécanisée ait été perçue comme un fardeau par les salariés, ces derniers ont néanmoins fait le choix historique de ne pas investi r dans le développement d'institutions visant à contrer cette stratégie.

Durant la même période, un modèle alternatif au fordisme se développait dans les usines d'automobiles britanniques. Retenons l'essentiel de l'exposé. L'influence des techniques de production américaines fut très important en Grande-Bretagne durant les années 1910 et 1920 , notamment à l'usine Ford de Manchester mais aussi de façon plus générale. Toutefois les constructeurs autres que Ford optèrent pour un modèle de contrôle managérial alternatif, reconnaissant la force des traditions de métier et laissant beaucoup de place à l'autonomie ouvrière. «British employers abandoned the direct control route of the Americans. Instead, control was to be achieved indirectly through incentive payment systems and self-regulation» (p. 110). Ce modèle britannique, issu d'un croisement entre les techniques modernes de production et l'autonomie ouvrière, donna de très bons résultats économiques entre les deux Guerres. Toutefois, selon l'auteur, il entraîna deux effets néfastes à long terme, à savoir le faible investissement en 
technologie et le retard dans le développement des méthodes de gestion, deux facteurs associés à ce modèle plaçant trop peu d'emphase sur le contrôle réel de la force de travail. À noter que cette dernière association constitue une pièce maîtresse dans l'analyse de Lewchuk. Cela l'amène à accorder, en pratique, un rôle primordial au niveau de l'atelier, voir à l'influence ouvrière, dans l'explication du déclin de l'industrie automobile britannique.

Le retard technologique aurait été pleinement senti par les entreprises britanniques après la Deuxième Guerre. Celles-ci auraient alors investi en technologie. Il s'ensuivit un profond déséquilibre entre ces nouvelles techniques de production et les institutions de production («production institutions»), cette contradiction étant exacerbée par l'autonomie croissante des délégués d'atelier. La formule suivante touche l'essentiel de la thèse: «The collapse of the British motor vehicle industry came when firms were forced to abandon the interwar strategy of labour intensive production techniques, self-regulation of effort norms and weak management control of the production process. The superiority of the American perfected automated production system forced British firms to shift to more capital intensive production techniques after World War II. The attempt to merge American style production techniques with British production institutions was a disaster from the employers' point of view» (p. 222).

Bien que cette thèse soit convaincante, elle incite néanmoins à certaines réserves, surtout sur le traitement de la période la plus récente. D'abord, il est possible que l'auteur exagère l'importance de l'implantation de la nouvelle technologie «américaine» après la Deuxième Guerre. Le matériel présenté au chapitre 9 ne démontre pas que la technologie ait été modifiée de façon aussi marquée durant la période 1945-1960. Cette réserve touche évidemment la thèse principale de l'auteur sur le déséquilibre entre les techniques de production et les institutions de production. En second lieu, ce même chapitre, peut-être trop bref, sur l'écroulement («the collapse») du système britannique entretient une certaine confusion entre des phénomènes et des périodes différentes. Par exemple, la crise des relations du travail qui atteignit son paroxysme à la fin des années 1960 et le déclin de la production automobile, surtout depuis dix ans, s'expliquent-ils de la même façon?

Une troisième réserve a trait au modèle théorique sur le contrôle du travail. L'auteur insiste beaucoup sur le nécessaire compatibilité entre la technologie et les institutions de production, lesquelles renvoient surtout au contrôle social du travail. Dans cette démarche, en pratique, l'auteur en vient à associer de très près la stratégie de contrôle direct (ou fordisme) à la qualité du contrôle managerial, voire à l'efficacité. Cette interaction est plus complexe. Plusieurs recherches récentes ont fait ressortir la complexité de cette relation entre les techniques de production et les différentes formes de contrôle du travail. Par exemple, l'auteur fait état de la stratégie de Edwardes à Longbridge, à savoir la combinaison d'une technologie de pointe au contrôle direct sur le travail. Or, en théorie, il n'est pas du tout démontré que cette combinaison soit optimale; et dans le cas de Longbridge, son succès paraît fort douteux. Même sur la base d'une importante documentation historique, la constitution d'un modèle théorique sur le contrôle du travail demeure donc problématique.

En définitive, j'ai donc beaucoup apprécié cet ouvrage, qui me semble de la plus haute qualité. L'auteur a développé, notamment par le travail en usine, une connaissance très riche de l'industrie de l'automobile. L'étude historique l'amène à soulever des questions fondamentales et à avancer une thèse claire et stimulante. Dans cette démarche l'auteur profite pleinement de l'étude comparée entre ces deux pays, laquelle s'avère particulièrement fructueuse.

Jacques BÉLANGER 